\title{
Content Validity and Reliability of the 13-Item Sense of Coherence Scale among 13-15 Year Old School Children in Chennai City
}

\author{
Pavithra $\mathrm{R}^{1}$, Lalithambigai $\mathrm{G}^{2}$, Mohammed Junaid ${ }^{2}$, Madan Kumar $\mathrm{PD}^{3}$
}

\section{ABSTRACT}

Objective: To assess the content validity and reliability of the 13-item Sense of Coherence (SOC) scale among 13-15 year old school children in Chennai city. Participants: A total of 258, 13 - 15 year old ethnic Dravidian students from two randomly selected schools within Chennai city, present on both days of the study were included. Study design: A cross sectional study. Method: The 13-item Sense of Coherence questionnaire was translated to the vernacular, back translated and subjected to expert opinion to check its validity. The 13-item SOC questionnaire was used to assess the Sense of Coherence of the selected school students. The questionnaire was re-administered after one week to measure to measure Internal consistency reliability and Infraclass correlation co-efficient. Results:_ The Internal consistency reliability checked using Cronbach $\alpha$ was found to be 0.784 . The intra class correlation co-efficient was 0.7 and remained the same with an item deleted. Conclusion: Our results suggest that the 13 item SOC questionnaire is a valid and reliable epidemiological tool among an adolescent Dravidian population in Chennai city. Further studies are recommended to assess the stability of SOC concept over a period of time.

Keywords: Sense of Coherence, Reliability, Content Validity, Children

The proverb, "He who has health has hope, he who has hope has everything" enunciates the importance of health in the life of an individual. Health - the fundamental right of human beings, over the decades has evolved from individual perspective to global well being. The concept of health is no more perceived from a biomedical point of view but is considered as a holistic model adapting to the changes and influences of the social, cultural, economical, environmental and political arena (Park K., 2009).

Twenty first century public health research has focused extensively on social determinants of health. Various theoretical models stressing the social context and its interaction with biological

\footnotetext{
${ }^{1}$ Postgraduate student, Department of Public Health Dentistry, Ragas Dental College and Hospital, Chennai.

${ }^{2}$ Senior lecturer, Department of Public Health Dentistry, Ragas Dental College and Hospital, Chennai

${ }^{3}$ Professor and Head, Department of Public Health Dentistry, Ragas Dental College and Hospital, Chennai (C) 2015 I Pavithra R, Lalithambigai G, Mohammed Junaid, Madan Kumar PD; licensee IJIP. This is an Open Access Research distributed under the terms of the Creative Commons Attribution License (http://creativecommons.org/licenses/by/2.0), which permits unrestricted use, distribution, and reproduction in any Medium, provided the original work is properly cited.
} 


\section{Content Validity and Reliability of the 13-Item Sense of Coherence Scale among 13-15 Year Old School Children in Chennai City}

and psychological factors have emerged (Freire et al., 2001). One of the most accepted theoretical model that has been propounded is the Salutogenic theory.

The theory of Salutogenesis (Aaron Antonovsky, 1987) elucidated the distinct factors that aid in the promotion of health as against the factors that modified or attempted to prevent specific disease. Salutogenic model is based on two concepts: General Resistance Resouces (GRR) and Sense of Coherence (SOC). General Resistance Resources concentrates on factors like social support, material resources, coping strategies and family socialization that neutralize the effects of stressful life events and promote successful tension management. Sense of Coherence, the central construct of the Salutogenic model, states that in order to promote well being, it is important for people to focus on their resources and capacity, rather than on their disease (Bonanato et al., 2009). Aaron Antonvosky postulated that people with higher SOC are able to utilize the available resistant resources from their surrounding and maintain health inspire of stressful situations.

The three components of SOC are: Comprehensibility - the ability of people to understand what is happening around them; Manageability - the extent to which they feel capable of managing the situation and Meaningfulness - the ability to find meaning in the situation (Lindstrom and Eriksson 2005).

A 29 item and 13 item Sense of Coherence questionnaire (SOC) was developed after profound interview with 51 people who recuperated remarkably after having experienced severe trauma due to inevitable consequences of health conditions (Aaron Antonovsky, 1987). The structure and properties of both the 29 item and 13 item SOC scale were validated in 1993 (Aaron Antonovsky, 1993).

Many studies have been carried out in the past to outline the influence of Sense of Coherence on general health, oral health and oral health related behavior (Ayo-Yusuf et al., 2008, Baker et al., 2010, Gururatna et al., 2014). A study conducted to assess the relationship between Sense of Coherence and dental attendance pattern reported that subjects with weak SOC were likely to visit the dentist only in the event of problem than those with stronger SOC (Freire et al., 2002). This revealed that SOC had a direct influence on oral health.

The 13 item SOC scale has been successfully used in 33 countries in 32 languages of various cultural backgrounds, including few Indian Languages (Khatri et al., 2014). Since the availability of a questionnaire in local language is a requisite for epidemiological research (Castro et al., 2008), this study attempts to check the content validity and reliability of the 13 item SOC scale among an ethnic Dravidian population of South India. 


\section{MATERIAL AND METHODS:}

A cross sectional study was undertaken to assess the content validity and reliability of the 13 item Sense of Coherence scale among an ethnic Dravidian population of a South Indian State. Ethical clearance to conduct the present study was obtained from the Institutional Review Board, Ragas Dental College and Hospital, Uthandi. From a total number of 15 zones under city limits as classified by the Corporation of Chennai, two zones (zone No.1 and zone No.9) were selected randomly for the present study. List of schools in the selected two zones were sourced from the Directorate of school education, Chennai. For the present study, only higher secondary and senior secondary schools offering both English and Tamil as the medium of instruction were selected. Out of 25 schools in zone 1, a senior secondary school (school one) and one higher secondary school (school two) out of 62 schools in zone 9 were selected. Permission to conduct the study involving the school students were obtained from the school authorities.

A total of 270 students, 146 from school one and 124 from school two were chosen as the study population. Students of $13-15$ years, who consented to participate and those present on both days of the study were included in the study. School records were verified to identify children of ethnic Dravidian background and those who belonged to this group were selected.

Tamil is the vernacular of Tamilnadu. Hence, the 13 item SOC scale was translated to the vernacular. The validity was checked by back translation method, involving blind retranslation into English. The validity of translation was verified by experts in both languages.

Data was collected through a self- administered 13-item SOC questionnaire which was distributed to the students during their school hours. Filled questionnaires were collected after 20-25 minutes and were checked for completeness. The questionnaire consisted of 13 questions, to be answered on a seven-point Likert scale, with five questions on comprehensibility, four on meaningfulness and four on manageability.

On day one, both the Tamil and English version of the questionnaire was administered to two hundred and seventy students who were present and their responses (T1) were collected. After a week, the questionnaire was re-administered to check the test-retest reliability. Students were advised to adhere to the procedure that was followed earlier when the questionnaire was administered for the first time. Two hundred and fifty eight responses (T2) were received - 138 from school one and 120 from school two. Twelve students were absent on day two and they were excluded from the study.

Assessment of intra class correlation co-efficient and internal consistency reliability was carried out to re-establish the psychometric properties of the 13 item SOC scale. Responses of T1 and T2 were collated and subjected to statistical analysis using SPSS 20.0 (Statistical Package for the Social Sciences for Windows; SPSS Inc., Chicago, IL, USA). The Internal consistency 
reliability was tested using Standardized Cronbach $\alpha$ co - efficient. Item - total statistics was calculated to check correlation between the questions and to check Cronbach $\alpha$ value when an item was deleted.

\section{RESULTS:}

The English version of the 13 item SOC scale was translated to Tamil back translated to English and was found to match with original English version. The content validity was checked by subject experts and was acceptable.

Table 1 shows the distribution of Cronbach $\alpha$ co-efficient for each question and ranged between 0.4 to 0.8 . The overall intra class correlation co-efficient was 0.784 between $\mathrm{T} 1$ and $\mathrm{T} 2$.

Question number 2, 10 and 11 reported a slightly lower Cronbach $\alpha$ value in the range of 0.4. Meanwhile, question number 4 and 7 reported a higher value of 0.8 and 0.7 respectively. The values of the other questions ranged between $0.5-0.6$.

The Cronbach $\alpha$ for the individual components of SOC, Comprehensibility, Manageability and Meaningfulness was $0.642,0.658$ and 0.548 respectively.

Table 2 shows the distribution of corrected - item total correlation value, which ranged between 0.1-0.5. The overall Cronbach $\alpha$ value remained 0.7 showing that the reliability of the questionnaire was not much affected when any item was deleted among this population.

\section{DISCUSSION:}

The purpose of this study was to translate the English version of the 13 item SOC scale to Tamil and check its reliability among 13-15 year old school children in Chennai city.

The Cronbach $\alpha$ value of the 13-item SOC scale ranged between $0.70-0.92$ and was found to be equally valid and reliable like the 29- item SOC, with a Cronabach $\alpha$ value between 0.700.95(Lindstrom and Ericksson., 2005). Since the 29-item scale demanded longer time duration, the 13-item scale was recommended.

In the present study, the translated version of the 13-item SOC scale was back translated to English and was found to match the original version. Further, the questionnaire was readministered after one week in order to ensure that the time interval was neither too short nor too long. To check the reliability of a questionnaire the interim period between the days of the administration of the questionnaire is important. If it is too short, memory skills will influence the response and yield confounding results. In case of longer time interval, the attribute being examined might get changed and low correlation may indicate this change rather than poor reliability (Lawshe 1975). 
Aaron Antonvosky stated that the concept of Sense of Coherence is in its developmental stage among adolescents. Dental health education has a small positive effect on oral health status (Kay EJ and Locker D). This necessitated researchers to identify psychosocial predictors of oral health (Baker et al., 2014). Ayo - Yusuf et al conducted studies among adolescents using 13 item SOC scale and found that their SOC scores had a direct influence on their oral health and oral health related behavior. Gururatna et al., (2014) reported that SOC had an influence on oral health and suggested that interventions designed to promote SOC may present an opportunity to enhance children's experience of oral health.

An interventional study among 10 - 11 year old school children in Thailand proved that improving the SOC of children in turn resulted in improved oral health status (Nammotri et al, 2013). Ayo- Yusuf studied the longitudinal association between SOC and tooth brushing among adolescents, using an integrated behavior therapy model. It was reported that this approach promotes better oral health. Results from the above mentioned studies delineate that; SOC can be used as a positive predictor for oral health among adolescents. Further, the 13 item SOC scale which was originally developed for adults can also be used for children of 12 years of age (Honkinen et al, 2006).

These advantages of the 13 item SOC scale necessitated the need for the development of a 13 item SOC scale in the local vernacular and to check its reliability among 13-15 year old school children in Chennai city so as to enable further studies in this field.

To the best of our knowledge, this was the first study that has attempted to check the internal and test retest reliability of the 13 item SOC scale among an ethnic Dravidian population of south India.

Various difficulties were reported in completing the questionnaire. Lee and Colleagues found that Japanese respondents reported difficulty with filling in the scale. The Chinese were reported to skip questions (Lindstrom and Eriksson. 2005). However in the present study, participants were comfortable filling in their responses on a seven-point Likert scale and no questions were skipped. In general, the participants found it difficult to understand question number two (Has it happened in the past that you were surprised by the behavior of people who you thought you knew well), question number 10 (Many people - even those with a strong character sometimes feel like sad sacks. How often have you felt this way in the past?) and question number 11 in the 13 item SOC scale.

In a systematic review regarding the validity and reliability of the SOC scale, the Cronbach $\alpha$ ranged from 0.70 to 0.92 (Lindstrom and Eriksson 2005). Our study reported a Cronbach $\alpha$ value of 0.784 which is acceptable. Though the participants found difficulty in answering question 
number 2, 10 and 11 of the 13 item SOC scale, the Cronbach $\alpha$ value remained 0.7 even with these items deleted.

The limitation of this study was that the results obtained from this study cannot be generalized to the adult population and no attempt was made to assess whether the intelligent quotient (IQ) of students influenced their ability to complete the questionnaire. More research is required to eliminate these limitations and to find whether there exists an association between SOC scale and gender among various populations.

\section{CONCLUSION:}

Our study revealed that the 13-item SOC scale is a valid and reliable tool to measure Sense of Coherence among an adolescent Dravidian population in Chennai city. Further studies are needed to assess the stability of SOC concept over a period of time.

\section{REFERENCES:}

Antonovsky, A. 1987. Unraveling the mystery of health. How people manage stress

and stay well. $1^{\text {st }}$ edn. San Francisco: Jossey-Bass.

Antonovsky, A. (1993): The structure and properties of the sense of coherence scale. Social Science and Medicine 36, 725-733.

Ayo-Yusuf, O.A., Reddy, P.S. and van den Borne, B.W. (2008): Adolescents' sense of coherence and smoking as longitudinal predictors of self reported gingivitis. Journal of Clinical Periodontology 35, 931-937.

Baker, S.R., Mat, A. and Robinson, P.G. (2010): What psychosocial factors influence adolescents’ oral health? Journal of Dental Research 89, 1230-1235.

Bonanato, K., Paiva, S.M., Pordeus, I.A., Ramos-Jorge, M.L., Barbabela, D. and Allison, P.J.(2009): Relationship between mothers' sense of coherence and oral health status of preschool children. Caries Research 43, 103-109.

Castro, R.A., Cortes, M.I., Leao, A.T., Portela, M.C., Souza, I.P.R., Tsakos, G., Marcenes, W. and Sheiham, A. (2008): Child- OIDP index in Brazil: Cross cultural adaptation and validation. Health and Quality of Life Outcomes 6, 68.

Freire, M., Hardy, R. and Sheiham, A. (2002): Mothers' sense of coherence and their adolescent children's oral health status and behavior. Community Dental Health 19, 24-31.

Freire, M.C.M., Sheiham, A. and Hardy, R. (2001): Adolescents' sense of coherence, oral health status and oral health related behaviours. Community Dentistry and Oral Epidemiology 29, 204-212.

Gururatana, O., Baker, S.R. and Robinson, PG. (2014): Determinants of children's oral health related quality of life over time. Community Dentistry and Oral Epidemiology 43, 206215. 
Honkinen, P.L., Suominen, S., Rautava, P., Hakanen, J. and Kalimo (2006): The adult sense of coherence scale is applicable to 12-year-old schoolchildren an additional tool in health promotion. Acta Pcediatrica 95, 952-955.

Khatri, S.G., Acharya, S. and Srinivasan, S.R (2014): Mother's sense of coherence and oral health related quality of life of preschool children in Udupi Taluk. Community Dental Health. 31, 32-36.

Kay, E.J. and Locker, D.(1996): Effectiveness of oral health promotion: A review. International Journal of Evidence based dentistry.2, 13-15.

Lawshe, C.H. (1975): A quantitative approach to content validity. Personal Psychology 28, 563575.

Lindstrom, B. and Eriksson, M. (2005): Validity of Antonovsky's sense of coherence scale: a systematic review. Journal of Epidemiology and Community Health 59, 460-466.

Nammontri, O., Robinson, P.G. and Baker, S.R.(2013). Enhancing oral health via sense of coherence: a cluster randomized trial. Journal of Dental Research 92, 26-31.

Park, K. 2009. Textbook of preventive and social medicine. 20th edn. P 12. Jabalpur: M/s Banarsidas Bhanot publishers.

Table 1.Distribution of Cronbach $\alpha$ value for each question in the 13-item SOC scale

\begin{tabular}{|c|c|c|}
\hline QUESTION & CRONBACH & $\alpha \quad \operatorname{MEAN}($ S.D) \\
\hline NO & VALUE & \\
\hline 1 & .67 & $10.37(2.47)$ \\
\hline 2 & .41 & 7.56(2.69) \\
\hline 3 & .68 & 7.81(3.21) \\
\hline 4 & .80 & $11.53(2.74)$ \\
\hline 5 & .64 & $9.5493 .00)$ \\
\hline 6 & .54 & $8.62(2.88)$ \\
\hline 7 & .71 & $9.56(2.82)$ \\
\hline 8 & .53 & $7.65(2.85)$ \\
\hline 9 & .56 & $7.86(2.92)$ \\
\hline 10 & .48 & $8.47(2.56)$ \\
\hline 11 & .43 & $10.63(2.39)$ \\
\hline 12 & .56 & $10.44(2.84)$ \\
\hline 13 & .58 & $9.85(2.67)$ \\
\hline $\begin{array}{l}\text { Intra class } \\
\text { correation co- } \\
\text { efficient }\end{array}$ & .784 & $119.89(17.14)$ \\
\hline
\end{tabular}


Content Validity and Reliability of the 13-Item Sense of Coherence Scale among 13-15 Year Old School Children in Chennai City

Table 2: Distribution of Item Total Statistics

\begin{tabular}{lll} 
Question no: & Corrected item total correlation & $\begin{array}{l}\text { Cronbach's alpha if item } \\
\text { deleted }\end{array}$ \\
\hline 1 & .112 & .697 \\
2 & .269 & .679 \\
3 & .286 & .677 \\
4 & .201 & .687 \\
5 & .409 & .658 \\
6 & .312 & .673 \\
7 & .272 & .678 \\
8 & .456 & .651 \\
9 & .449 & .652 \\
10 & .381 & .663 \\
11 & .276 & .678 \\
12 & .295 & .675 \\
13 & .336 & .670
\end{tabular}

